\title{
Quality of health care with regard to detection and treatment of mental disorders in patients with coronary heart disease (MenDis-CHD): study protocol
}

\author{
Samia Peltzer ${ }^{1^{* \dagger}}$ (D) Hendrik Müller ${ }^{2 \dagger}$, Ursula Köstler ${ }^{3}$, Katja Blaschke $^{4}$, Frank Schulz-Nieswandt ${ }^{3}$, Frank Jessen ${ }^{2,5}$,
} Christian Albus ${ }^{1}$ and on behalf of the CoRe-Net study group

\begin{abstract}
Background: Mental disorders (MD), such as depression, anxiety, and cognitive impairment, are highly prevalent in patients with coronary heart disease (CHD). Current guidelines on cardiovascular diseases recommend screening and appropriate treatment of MD; however, the degree of implementation of such recommendations in clinical practice is unknown. This study aims to analyze the quality of health care of patients with CHD and MD. Specifically, we aim to analyze (1) the quality of care, (2) trajectories of care, and (3) barriers regarding the detection and treatment of MD. Moreover, we want to identify potentials of changes in health care delivery towards more patient-centered care. The results of this study shall be the first step towards value-based care of people with $\mathrm{CHD}$ and comorbid mental disorders.
\end{abstract}

Methods: We aim to include the following participants: adult patients with CHD $(n=400)$, their relatives $(n=350)$ and physicians $(n=80)$. A particular focus will be on the vulnerable subgroups of patients with CHD and congestive heart failure (left ventricular ejection fraction $<40 \%$ ) and on the underrepresented group of women with CHD. We will apply a mixed-method approach with a quantitative and a qualitative part.

Patient-related outcomes (e.g., health-related quality of life, needs, and preferences regarding health care, reasons for non-detection, and lack of treatment of MD) will be explored in a multi-perspective approach including patients, relatives, and physicians' perspectives. Furthermore, routine data from four statutory health insurance funds (SHI) will be analyzed regarding the frequency and treatment of MD in CHD patients.

Discussion: MenDis-CHD will provide important insights into the trajectories of health care, quality of health care, barriers, patient needs and preferences as well as expectations and satisfaction with health care in patients with CHD and MD. Potential implications of MenDis-CHD are to enable health care providers to redesign care pathways concerning the treatment of mental comorbidity in patients with CHD by proposing value-based changes in health care and by understanding the barriers to and facilitators of change towards patient-centered care.

Trials registration: German clinical trials register (Deutsches Register Klinischer Studien, DRKS) ieRegistration Number: DRKS00012434, date of registration: May 11th, 2017.

Keywords: Coronary heart disease, Mental disorders, Cognitive impairment, Value-based health care

\footnotetext{
*Correspondence: Samia.peltzer@uk-koeln.de

†Samia Peltzer and Hendrik Müller are co-first authors.

'Department of Psychosomatics and Psychotherapy, University of Cologne,

Faculty of Medicine and University Hospital Cologne, Kerpener Straße 62,

50937 Cologne, Germany

Full list of author information is available at the end of the article
}

(c) The Author(s). 2019 Open Access This article is distributed under the terms of the Creative Commons Attribution 4.0 International License (http://creativecommons.org/licenses/by/4.0/), which permits unrestricted use, distribution, and reproduction in any medium, provided you give appropriate credit to the original author(s) and the source, provide a link to the Creative Commons license, and indicate if changes were made. The Creative Commons Public Domain Dedication waiver (http://creativecommons.org/publicdomain/zero/1.0/) applies to the data made available in this article, unless otherwise stated. 


\section{Background}

Coronary heart disease (CHD) is the leading cause of morbidity and mortality in Europe and the USA [1, 2]. In 2008, approximately 17 million people died worldwide from $\mathrm{CHD}$, and $10 \%$ of the burden of disease worldwide is caused by CHD [3]. Due to advances in the acute treatment of CHD, short-term mortality has decreased, but morbidity on a population level has increased $[1,2,4]$. In recent years, gender aspects concerning $\mathrm{CHD}$ received increasing attention. Men have a twofold higher CHD prevalence $(12.3 \%)$ than women $(6.4 \%)[4,5]$. However, there is a stronger increase in incidence in older women compared to men [4]. CHD-related mortality in women also appears to be slightly higher [5]. Several reasons have been discussed, including the older age of women at the acute cardiovascular event, but also lower detection rates and less treatment of cardiac symptoms [5, 6]. Also, mental disorders (MD) have been found to be highly prevalent among patients with CHD, particularly in women [7]. Especially depression, anxiety disorders, cognitive impairments [8] and other MDs affect approximately $50 \%$ of all CHD patients $[7,9,10]$.

MD - whether they are pre-existing conditions or consequences of CHD - act as particularly strong barriers to treatment adherence and impede efforts to lifestyle change [1], resulting in an approximately two-fold greater morbidity and mortality risk in CHD $[1,7,9,11,12]$.

In detail, several mechanisms are discussed: first, unhealthy lifestyle (e.g., smoking, high alcohol consumption) is more prevalent in patients with CHD and MD. Second, CHD-patients with MD are more resistant to behavior change and have low medication adherence. Third, psychobiological mechanisms are found, which increased risk for CHD, even if the 'classical' risk factors are controlled for (e.g., alterations in autonomic functions, in the hypothalamic-pituitary axis and in inflammatory markers [13]). The prevalence of depressive symptoms in CHD patients is approximately $31 \%$ in women and $20 \%$ in men. Anxious symptoms occurred approximately in $39 \%$ of the women and in $22 \%$ of the men 1.4 years after hospitalization for CHD [14]. Consequently, recent, national and international guidelines on primary and secondary prevention of CHD recommend routine screening for $\mathrm{MD}$ and adequate treatment for at-risk patients $[1,10,15]$.

Given that adherence to CHD guidelines in physicians is generally low, even when these guidelines predominantly comprise somatic recommendations [16], it is unlikely that general practitioners and cardiologists regularly screen for MD due to typical restraints such as a lack of time and low reimbursement of verbal interventions [12, 17]. However, a systematic review in 2013 showed that depression screening is only beneficial for identifying depressed patients that were not already diagnosed or treated for depression. Also, the accuracy of screening tools seems to be exaggerated by the inclusion of already diagnosed patients and the selective reporting of results from cut-off scores. Following this review, a wide range of routine screening would entail high costs and increase the number of patients using antidepressants. Hence, it is important to not only look at the screening for MD and cognitive impairements but also to look at diagnostic and treatment following the screening [18]. Overall, the status of quality of care in CHD patients with comorbid mental disorders is unknown. Moreover, it is unclear what the trajectories of care of patients with CHD and cognitive impairement are.

In addition, no data on personal preferences, needs, and expectations of patients with CHD and their relatives regarding the detection and treatment of MD have been published. Thus, there is an urgent need to explore the current state of care in patients with CHD and MD to identify relevant factors that are preventing improved care in health care systems.

\section{Methods \\ Aims}

The study examines [1] the current quality of health care regarding to the detection and treatment of $\mathrm{MD}$ in patients with CHD, [2] the experiences of physicians in treating their patients according to guideline, [3] needs and preferences of the patients and [4] possible barriers for the implementation of guideline-based diagnostic and treatment.

Further, routine data from health insurance funds (SHI), collected continually for reimbursement and stored for several years by health insurers, will be allocated [19]. The SHI-data will be analyzed concerning frequency of CHD as well as sex and age distribution, use of the resource, costs, and trajectories of care (e.g., diagnosis of MD, psychotropic medication, psychotherapy, hospital stay, sick leave certificates, and early retirement).

The findings of the MenDis-CHD will contribute to an overview of the current state of health care in CHD with the aim of improving and modifying care delivery, so that appropriate interventions ensure value-based health care.

\section{Theoretical framework and research platform}

MenDis-CHD is located in Cologne, Germany and is one of three current projects of the 'Cologne Care Research and Development Network' (CoRe-Net). Core-Net is funded by the Federal Ministry of Education and Research (BMBF), and the MenDis-CHD study protocol was subject to peer review by the funding body before approval. The value-based health care concept by 
M.E. Porter [20-22] forms the framework of CoRe-Net, which has the aim to create a data-driven learning environment employing research and practice to improve health and social care organizations by transforming them into a system that develops and delivers care with greater value. We define value-based health care as cost-consciously redesigning care processes and structures according to the needs of patients, which includes the two dimensions of patient-related and economic aspects. For further details about CoRe-Net see [23].

\section{Participants of MenDis-CHD}

Overall, we aim at recruiting a total number of $N=830$ participants. The total sample includes subsamples of patients, relatives, and physicians. Since Jan 15, 2018, the MenDis-CHD study is in the recruitment phase.

\section{Patients}

We intend to enroll adult patients ( $n=400 ; 50 \%$ women) with angiographically documented CHD treated for stable angina pectoris, acute coronary syndromes, percutaneous coronary intervention, or bypass surgery. Participants must be able to give informed consent and have sufficient German language skills. Exclusion criteria are severe or instable physical or mental conditions (e.g., severe illnesses such as cancer, acute suicidal ideation, delirium, and moderate to severe dementia). Since we are conducting a descriptive and exploratory study, but not a confirmatory one, we did not performed a sample size calculation based on a power calculation. Rather the focus of MenDis-CHD is on vulnerable subgroups. These vulnerable subgroups comprise (1) older female CHD-patients which were underrepresented in past studies, (2) CHD-patients with comorbid mental disorders and/or mild cognitive impairment (expected prevalence of MD and MCI is 30-50\%), and (3) CHD-patients with congestive heart failure (expected prevalence of $30 \%$ with a left ventricular ejection fraction of $<40 \%$ [24]). To obtain a realistic estimate of the health care situation, patients will be recruited in cardiology departments of hospitals, cardiology practices, and rehabilitation clinics.

Thus, the rational for the intended sample size are this estimated frequency of the gender distribution, mental and cognitive comorbidity and patients with congestive heart failure as well as the goal of recruiting in different sectoral areas (hospitals, rehabilitation clinics, and cardiology practices). By this rationale, we aim at a sufficiently large sample sizes to perform the statistical comparison in these vulnerable subgroups. In sum, the recruitment of a total $N$ of 400 patients is planned. Thus, we aim to recruit $n=200$ women, $n=130-200$ patients with MD or MCI and $n=120$ with congestive heart failure.
All 400 patients (with and without MD) will take part in the quantitative study. $N=20$ participants of the patient sample will be invited to join qualitative interviews.

\section{Relatives}

We aim at recruiting a total number of $n=350$ relatives of the CHD patients for the quantitative survey. Of these, $n=20$ will be asked to participate in qualitative interviews. The group of relatives is defined as every close person living in the household of the patients. Inclusion criteria for relatives are: 18 years of age or older, able to give informed consent and sufficient German language skills. Exclusion criteria are severe or instable physical or mental conditions.

\section{Physicians}

A total number of $n=80$ physicians (general practitioners (GPs), cardiologists, physicians at rehabilitation clinics, and psychotherapists) will be tried to be recruited for a quantitative assessment. $N=20$ physicians of this sample will also participate in qualitative interviews. Moreover, $n=40$ physicians will be randomly selected to participate in focus groups. Four focus groups, each consisting of ten participants will be conducted, including following specialties named above.

Please refer to Fig. 1 for details on the recruitment procedure and sub-samples.

\section{Study population of SHI data}

SHI data of patients who are inhabitants of Cologne continually insured between 2011 and 2012 and continually insured or deceased in 2013 through 2015 in one of the four participating health insurance companies - (estimated about 270,000 persons) will be provided.

\section{Assessments}

The design of MenDis-CHD is a monocentric cross-sectional mixed methods approach [25] comprising quantitative (primary and SHI data as secondary data) and qualitative research (interviews and focus groups).

\section{Quantitative studies}

Disease severity We assess disease severity of CHD by ascertaining the reason of admission to hospitals, cardiology practices, and rehabilitation clinics, the actual therapy, secondary diagnoses, cardiovascular risk factors, left ventricular ejection fraction, NYHA (New York Heart Association) and actual medication. If applicable, we assess severity of cardiac events (e.g., heart attack), congestive heart failure, bypass-surgery, percutaneous coronary intervention, cardiac valve surgery, echocardiography, cardiac catheterization. 


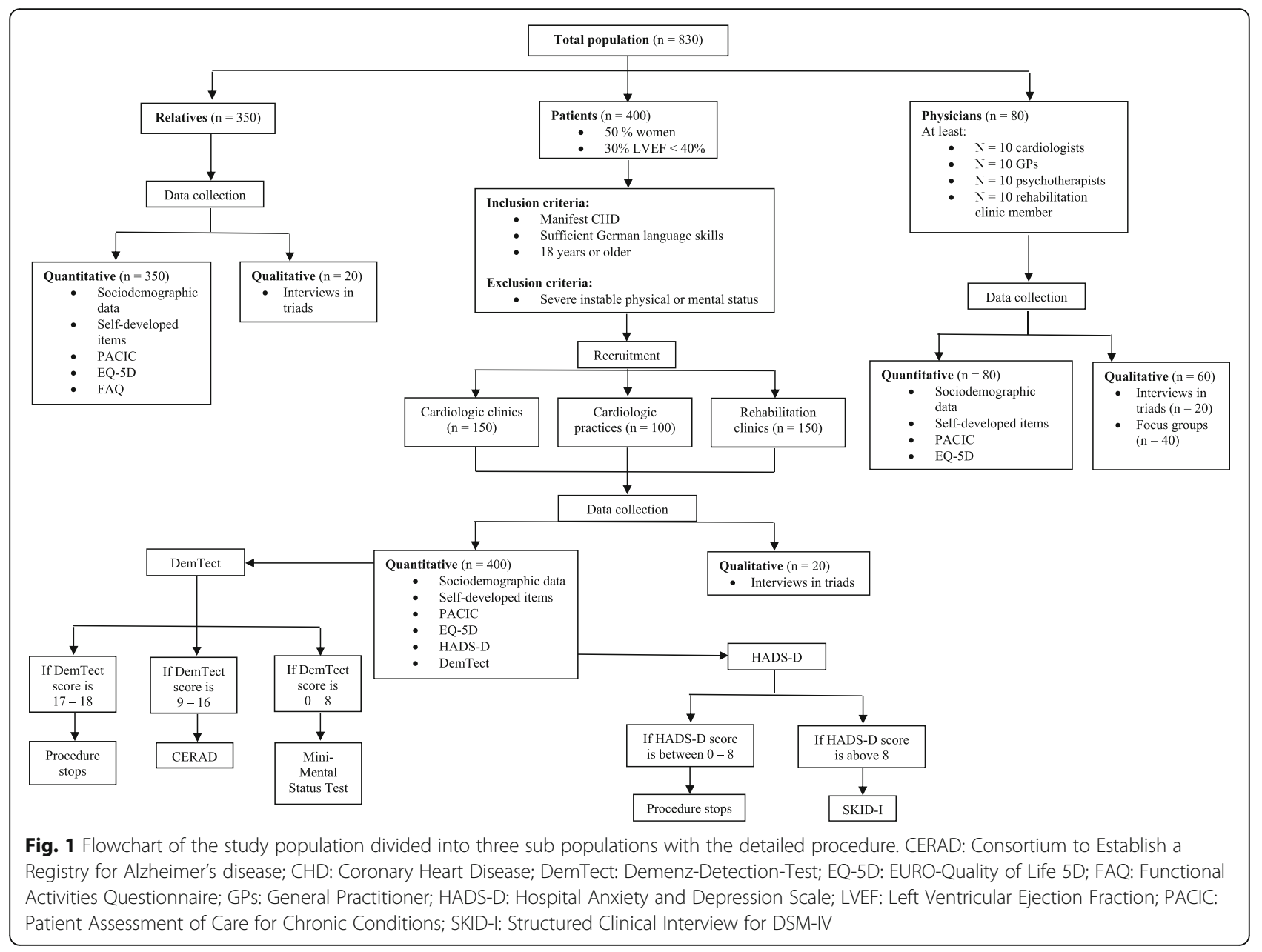

Clinical questionnaires The Hospital Anxiety and Depression Scale (HADS; [26, 27]) will be used as a screening tool for MD. The HADS is a self-report questionnaire to assess symptoms of depression and anxiety. It contains 14 items, which can be rated on a 4-point Likert-scale. Depression and anxiety sub-scales can be extracted. An anxiety/depression score between 0 and seven is considered 'negative', between eight and ten 'sub-syndromal positive' and between 11 and 21 'positive' for depression and/or anxiety. The reliability for the anxiety scale is $\alpha=.80$, for the depression scale $\alpha=.81$. The retest-reliability within 2 weeks is $\alpha=.80$ for the anxiety scale and $\alpha=.83$ for the depression scale. The construct validity is between $\alpha=.06-.08$. Cronbach's Alpha of the HADS is 0.80 , and the retest is indicated as $\alpha=.80$ [28].

The sensitivity and specificity for case detection are around .80 for both scales [28]. HADS-screening will be defined as 'positive' if the score is greater or equal to eight. In this case, the Structured Clinical Interview for DSM-IV (SKID-I; [29]) will be conducted. The SKID-I is a semi-structured interview to assess psychiatric symptoms and disorders as defined in the DSM IV
(Diagnostic and Statistical Manual of Mental Disorders; [30]). Lifetime and current diagnosis are assessed for the following disorders: [1] affective disorders, psychotic disorders, disorders through psychotropic substances, anxiety disorders, somatoform disorders, eating disorders and adjustment disorder. The test-retest reliability relevant for this study are within the range of $\alpha=.61-.76$ for unipolar affective disorders and $\alpha=.65-.63$ for anxiety disorders [31]. The sensitivity for affective disorders is .53 and for major depression .84. The specificity for affective disorders is .97 and for major depression .91 [32].

The Demenz-Detection-Test (DemTect; [33, 34]) will be used as a screening instrument to assess cognitive impairments. The DemTect contains five tests (1) a word list with immediate recall, (2) a number transcoding task, (3) a word fluency task, (4) a digit span reverse, and (5) a delayed recall of the word list. The total score of the DemTect gives an estimate whether the cognitive performance of the participant is normal for age (13-18 points), or whether mild cognitive impairment (MCI, 912 points) or dementia (8 points or below) should be suspected. In this study, we defined a DemTect score 
between 9 and 16 points as potentially indicative of cognitive impairments and thus will be regarded as 'positive'. In case of a positive screening with the DemTect, the Consortium to Establish a Registry for Alzheimer's Disease test battery (CERAD-Plus [35]) will be applied. If the DemTect score equals eight or is lower, the MiniMental State Examination (MMSE, 36) to detect significant cognitive deficits will be conducted. The test-retest reliability and the construct validity is above $\alpha=.80$. The sensitivity is .97 and specificity is .93 [34]. The CERAD-Plus is a neuropsychologic test battery for the diagnostic of dementia [35]. It contains ten sub tests, which assess cognitive performance in different cognitive domains: (1) verbal fluency, (2) Boston naming test, (3) Mini-Mental Status Examination, (4) word list learning, (5) constructional praxis, (6) word list delayed recall, (7) word list recognition and discriminability, (8) constructional praxis delayed recall, (9) Trail Making Test A and $B$, and (10) phonematic fluency. The raw data will be $\mathrm{z}$-standardized and adjusted for age, gender and education. If the $\mathrm{z}$-score is between -1.0 and $-1.5 \mathrm{SD}$, the cognitive performance is considered mildly impaired and below -1.5 as severe impaired.

The Mini-Mental Status Examination [36] is a screening instrument to assess signs of dementia. The maximum is 30 points. The scores are interpreted as follows: 30-27 point: no evidence of cognitive impairment, 2620 points: indicative of mild dementia, $19-10$ points: indicative of moderate dementia, nine points and below: indicative of severe dementia.

The test-retest reliability is $\alpha=.89$, and the construct validity is comparable to the construct validity of the DemTect [37]. The sensitivity is .88 , and the specificity is .86 [38].

To estimate functional abilities in cognitively impaired patients, all participating relatives are asked to fill out the Functional Activities Questionnaire (FAQ; [39]). The FAQ contains ten items and rates the patient's ability to perform daily activities. Participants rate each item on a 4-point Likert-scale ranging from $0=$ 'normal' to 3 $=$ 'dependent'. The scores are added to a sum score of 30. If the score equals nine or is higher an impaired function in daily activities is indicated. The construct validity is $\alpha=.847$, the sensitivity is $\alpha=.803$ and the specificity is $\alpha=.870[40]$.

Health care questionnaires Quantitative research in CHD patients, relatives and physicians will assess patients' trajectories and quality of care, barriers to guideline-based care, health care preferences, quality of life, the presence of MD, disease severity and provided health care.

The three versions of the health care questionnaire comprise in total 158 items for patients, 147 items for relatives, and 76 items for physicians. Questions are for example: 'Do you communicate with your physician (e.g., GP or cardiologist) about mental health issues?'

Furthermore, specific interventions and quality of health care in the enrolled patients are assessed by the Patient Assessment of Care for Chronic Conditions (PACIC; [41]). The PACIC contains 26 items, which are each rated on a 5-point Likert-scale ranging from $1=$ 'almost never' to 5 = 'almost always'. The higher the PACIC score, the better the patient-centeredness in health care from the patient's point of view. Cronbach's Alpha of the PACIC is 0.93, and the retest is indicated as $\alpha=.58$ [41].

The EURO-Quality of Life 5D questionnaire (EQ-5D; [42]) measures the health-related quality of life in five dimensions (mobility, self-care, usual activities, pain/discomfort, anxiety/depression) with three items per dimension. The items are rated on a 3-point Likert scale from $1=$ 'no problems' to $3=$ 'extreme problems.' The higher the score, the worse is the health-related quality of life. The test-retest reliability for the five sub scales are $\alpha=.69, \alpha=.77, \alpha=.64, \alpha=.48$, and $\alpha=.61$, respectively. All five sub scales are summed up in the life-quality index with a test-retest reliability of $\alpha=.75$. The visual analog scale is a $20 \mathrm{~cm}$ vertical visual scale with endpoints 'the best health condition you can imagine' and 'the worst health condition you can imagine.' The visual analog scale can be used as a quantitative individual measure of health with a test-retest reliability of $\alpha=92$ [43].

Questionnaires for physicians All professional health care providers $(n=80)$ will be quantitatively surveyed in cooperation with the OrgValue project of CoRe-Net. OrgValue (Organization \& Value) focusses on patientcenteredness and on economic aspects (resources, costs, payment). OrgValue analyzes the health care organizations involved in the care of patients studied in MenDis-CHD using a structured questionnaire to assess their knowledge, attitudes, and experiences concerning patient needs and preferences and barriers associated with MD detection and treatment.

Statutory health insurance data (SHI) Secondary claims data will be provided by four major health insurances of the state of North Rhine-Westphalia in Germany, from 2011 to 2015, for insuree living in Cologne. This SHI data is part of the CoRe-Net-database and will be used for this project (https://www.core-net. uni-koeln.de/index.php/de/core-net-datenbank/). Besides master data (e.g., age, sex, insurance status and period of insurance) information concerning the use of all sectors of care (inpatient and outpatient care, drug prescription, benefits in kind, long-term care) will be available and connectable by a non-identifiable study number. In 
detail, ICD-10 coded diagnoses from out- and inpatient care, medical services according to the EBM-Code (German physician fee manual), hospital stays with length of stay and OPS-procedures (Operations and Procedures Key), drug prescription with pharmaceutical registration number and linkage to ATC-Code (Anatomical Therapeutic Chemical Classification System) and DDD (Defined Daily Dose Classification), as well as information concerning inability to work (diagnosis, duration) and utilization of long-term care will be provided. For all services, provided SHI cost data will be available. Spatial data (e.g., INKAR: Indicators and maps for areaand town development) could be added.

In a first step, quality and plausibility checks of the SHI-data are performed. In a second step, patients with CHD and MD are identified by their diagnoses, which will be internally validated [44]. Inclusion criteria for CHD patients will be a hospital discharge diagnosis of CHD (ICD 10-GM code I20- I25), for chronic heart failure patients (ICD 10-GM code I11.0, I13.0, I13.2, I50), an outpatient diagnosis in at least two quarters of a or a hospital discharge diagnosis and for MD-patients (ICD 10 GM-code F00-F99, and more specific: F06.7, F32, F33) in one quarters of year or a respective hospital diagnosis. We will apply a cross-sectional design for basic epidemiological data (e.g., frequency of $\mathrm{CHD}$, CHD, and MD, psychotherapy, mortality) and a cohort design for the analysis of care trajectories starting in 2013, allowing a pre-observation period and a follow up of at least 2 years each.

These SHI-data will enrich the empirical part of the study [19] [45] as it allows to compare characteristics of general CHD-population with MD identified in the data to those included into the study and to determine the possible selection bias.

\section{Qualitative studies}

The qualitative module is designed to build on the questions of the quantitative module. With regards to those questions, the research design provides for data collection by interviews based on a predefined guideline. Guiding questions are for example: 'How are the patients experiencing health care?', 'In how far is the practitioners' advice complied with and to what extent can patients cope with their condition?' The coping of the relatives with regard to the patients disorder will be also assessed.

Focus groups Regarding the focus groups, the interpretations of the involved medical professionals will be analyzed [46]. It is of interest whether medical professionals are oriented towards national and international guidelines on primary and secondary prevention of CHD, especially the recommended routine screening for MD
$[1,9]$. The chosen procedure serves to inform the following face-to-face interviews.

Interviews in triades Secondly, we plan to perform 60 face-to-face interviews - selected as triads, each of those with a patient, his or her relatives, and the responsible practitioner.

Please refer to Fig. 2 for details on the recruitment procedure and subsamples of the qualitative part of the study.

\section{Procedures}

The University Hospital of Cologne (Department of Psychosomatics and Psychotherapy and Department of Psychiatry and Psychotherapy) is the only recruiting site. However, participants will be recruited in cardiology departments (University Hospital Cologne, Department of Internal medicine, Cardiology, Pneumology and Internal Intensive Care Medicine; Cologne-Merheim, clinics of the city of Cologne, Department of Cardiology, Rhythmology and Internal Intensive Care Medicine), patients from two cardiologic rehabilitation clinics (Clinic Roderbirken - Rehabilitation Centre for Heart and Circulatory Diseases; AmKaRe Cologne: out-patient cardiological rehabilitation centre) and from three cardiologic practices (Practice for Internal Medicine, Cardiology, Pneumology, Cologne, Wiener Platz 1; Practice for Cardiology Cologne, Josef-Haubrich-Hof 5; Practice for Cardiology Cologne, Wehrtmannstr. 1b). All patients screened for eligibility will be documented in a screening log. Patients who fulfill all inclusion and no exclusion criteria and provide written informed consent will be handed out the quantitative questionnaires and will be asked to participate in the qualitative interviews. If this screening with the HADS and/or the DemTect is positive, a second appointment will be arranged to perform the SKID-I and/or the CERADPlus. All participating researchers (SP and HM) were trained in applying the SKID-I and CERADPlus and are experienced in conducting the interviews with patients and research participants. The subsample of 20 participants, 20 relatives, and 20 physicians will be randomly contacted for a qualitative interview at a third time point. Furthermore, focus groups with 40 physicians will be randomly contacted.

\section{Outcomes}

\section{Quantitative}

The research questions of the quantitative module are: (1) Are actions for MD detection and treatment taken? Are these actions consistent with national and international guidelines on primary and secondary prevention of CHD? (2) What are the experiences of GPs and cardiologists who treat their CHD patients according to the 


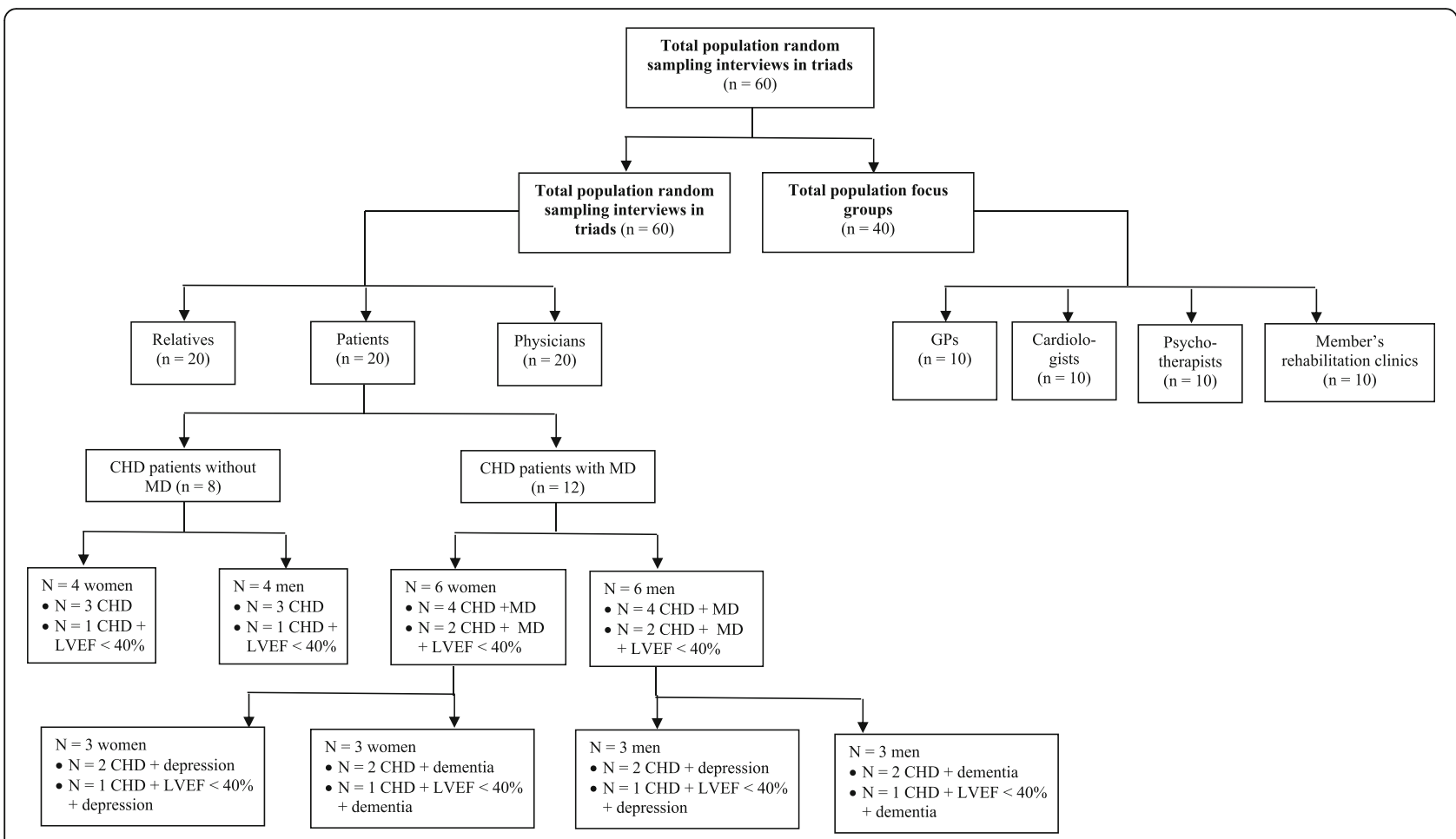

Fig. 2 Flowchart of samplings for interviews and focus groups. CHD: Coronary Heart Disease; GPs: General Practitioner; LVEF: Left Ventricular Ejection Fraction; MD: Mental Disorders

guidelines? For those GPs and cardiologists who do not adhere to the guidelines, what are the underlying reasons? (3) Do the assessment and treatment of MD correspond to CHD patients' needs and preferences? (4) What kind of treatment is offered for CHD patients diagnosed with MD? Are patients supported in finding the appropriate treatment? For what reasons do patients reject offered services or are not satisfied with them? (5) What are the barriers for a correct implementation of guideline-based diagnostic and treatment? What changes do the GPs and cardiologists suggest?

\section{Qualitative}

The qualitative module aims to analyze profoundly the patients' needs, preferences, attitudes, and barriers regarding value-based care of $\mathrm{CHD}$ patients with comorbid MD. The overall research question of the qualitative module focuses on the trajectories of care and quality care of the CHD-patients, relatives, and physicians. In how far are their expectations met and which barriers are they facing?

Overall, outcomes of the patients' data are: (1) prevalence of MD, (2) types of diagnostic procedures and treatment received, (3) quality of life, (4) satisfaction and short comings with respect to trajectories and quality of health care, and (5) expectations and needs with regard to health care (patient preferences). The outcomes will be analyzed separately for age groups and gender.
Main outcomes for the relatives' data are: (1) frequency of contact with the health care system and provided care, (2) caregivers' burden, (3) quality of life, (4) satisfaction with respect to trajectories and quality of health care, (5) patterns of perception about value, and (6) expectations and needs with regard to health care (preferences of relatives).

Main outcomes of the health care professionals' are: (1) knowledge, attitudes, and experiences concerning guideline recommendations, (2) personal views and experiences regarding MD detection, treatment, and value for the patient, and (3) limitations and barriers in the health care system. Further, outcomes of the focus groups of the health care professionals are about obstacles about guideline adherence, diagnosis and treatment of MD in CHD.

In the SHI data the outcomes of interest are prevalence and incidence of MD diagnosis in CHD patients, documented diagnostics, and treatments (e.g., prevalence of psychotropic medication and/or psychotherapy), documented costs according to sectors of care, as well as treatment persistence, frequency and duration of hospital stay, sick leave certificates, early retirement and death.

\section{SHI data}

We will provide frequency measures for patients with CHD in general and for those with (1) MD present 
before the hospital stay and (2) MD documented for the first time, i.e. no hint for MD by diagnosis or drug prescription in the interval of one respective two years before index stay (the MD group will be of main interest). This respective hospital stay serves as an index period, which allows the description of pre and post use of health care services and care trajectories. Besides estimating prevalence for $\mathrm{CHD}$ and $\mathrm{CHD}$ with comorbidity like MD, the data allows to assess by whom and how patients are treated (specialty of physician group, diagnostic procedures, non-medical therapy, drug prescribing, further hospital stays) and how patients comply with the treatment (adherence to medication regime according to the PDC methodology expressing the percentage of days covered with medication $[47,48])$. Absent days at work, early retirement and medical costs will also be analyzed. Predictors for MD treatment related to the information available in claims data will be assessed.

\section{Analysis}

\section{Statistical analysis}

Analysis of the quantitative data will include descriptive statistics, exploratory analysis (e.g., regression analysis), sub-group analysis and multivariate analyses to identify predictors of features of quality of health care. Therefore, statistical analyses will be exploratory and not confirmatory. Descriptive and analytic statistics will also be used for the analysis of the SHI data [49].

\section{Content analysis}

Qualitative data will be subject to content analysis. By using methods of qualitative social research, the study intends to demonstrate effects and relations using an exploratory approach and build on the interpretative method of Rosenthal [50].

\section{Discussion}

Data will be generated from multiple sources, including claims data, surveys, interviews and focus groups of professionals, patients, and relatives. Thus, our study design adopts a multi-perspective approach, which combines patients, their relatives and physicians views with in-depth analysis of trajectories, quality of health care, needs and preferences by quantitatively and qualitatively methods. Besides, we are able to gain insight into the general treatment of a non-selective population with CHD and MD by using SHI data.

The value-based concept by Porter [20-22] forms the analytical framework for our project. Consequently, our vision is to create value for the patient paying attention to both quality of health care and costs. MenDis-CHD will provide essential insights into the trajectories of health care, quality of health care, barriers, patient needs and preferences as well as expectations and satisfaction with health care in patients with CHD and MD.

The multi-method and multi-perspective approach of MenDis-CHD will provide data-driven analysis tools to enable care providers to redesign care pathways by proposing value-based changes in care and to understand the barriers to and facilitators of change towards patient-centered care. Restructuring complex care for vulnerable CHD patients is very much needed since there is evidence that these patients are underserved and often get lost in the transition between multi-professional and multi-institutional care providers [51, 52]. This generates unnecessary costs, ill health and thus low value for the patient.

MenDis-CHD has already begun to perform workshops inviting our scientific and practice partners, stakeholders, caregivers, patient and relative representatives to develop specific deliveries and methods for value-based health and social care in highly vulnerable patients with CHD and MD. Moreover, we have built up a representative network of recruitment centers. However, practice will have to show how well we succeed in recruiting patients against the backdrop of a significant reduction in inpatient stays. As already noted, women represent a vulnerable subgroup underrepresented in previous studies. Thus, recruitment will have to additionally show how demanding it is to achieve the targeted sample sizes of female patients.

The patient-centered products could be (a) an improved model of value-based health care for patients with CHD and MD (new standardized pathway with safe transitions), (b) a generalizable approach for transforming this model to other somatic patient groups with mental comorbidities and (c) gender-specific prompt sheets for patients.

Relatives-centered products may be (a) training units for relatives to enhance their ability to co-manage the process of comorbidity health care and (b) gender-specific prompt sheets for relatives. Professional-centered products could be (a) recommendations to improve professional cardiological guidelines and (b) trajectory-related directories as a coordination tool. The organization-centered products are self-analysis tools to raise awareness about mental comorbidities. Ultimately, we aim to improve the health care of people with somatic and mental disorders.

\footnotetext{
Abbreviations

ATC: Anatomical Therapeutic Chemical Classification System;

BMBF: Bundesministerium für Bildung und Forschung (English: Federal Ministry of Education and Research); CERAD: Consortium to Establish a Registry for Alzheimer's disease; CHD: Coronary heart disease; CoReNet: Cologne Care Research and Development Network; DDD: Defined Daily Dose Classification; DemTect: Demenz-Detection-Test; DSM IV: Diagnostic and Statistical Manual of Mental Disorders; EQ-5D: EURO-Quality of Life 5D; FAQ: Functional Activities Questionnaire; FHS: Faculty of Human Sciences; FM: Faculty of Medicine; FMESS: Faculty of Management, Economics and Social Sciences; GP: General Practitioner; HADS-D: Hospital Anxiety and Depression Scale; ICD-10: International Statistical Classification of Diseases
} 
and Related Health Problems, Version 10; IMVR: Institut für Medizinsoziologie, Versorgungsforschung und Rehabilitationswissenschaft (English: Health Services Research and Rehabilitation Science); INKAR: Indikatoren und Karten zur Raum- und Stadtentwicklung (English: Indicators and maps for area- and town development); LVEF: Left Ventricular Ejection Fraction; LYOL-C: Last Year Of Life in Cologne; MCl: Mild Cognitive Impairment; MD: Mental Disorders; MenDis-CHD: Mental Disorders in coronary heart disease; MMSE: Mini Mental State Examination; NYHA: New York Heart Association; OPS: Operations and Procedures Key; OrgValue: Organization \& Value; PACIC: Patient Assessment of Care for Chronic Conditions; PDC: Percentage of days covered (measure for adherence); SHI: Statutory Health Insurance Data; SKID-I: Structured Clinical Interview for DSM-IV; UHC: University Hospital Cologne; UoC: University of Cologne; ZVFK: Zentrum für Versorgungsforschung Köln (English: Center for Health Services Research Cologne)

\section{Acknowledgments}

We would also like to acknowledge the support from the further members of the CoRe-Net Co study group: Jun-Prof. Dr. Lena Ansmann, Institute of Medical Sociology, Health Services Research and Rehabilitation Science (IMVR), Faculty of Human Sciences (FHS), University of Cologne (UoC); Dr. Nadine Scholten and Dr. Ute Karbach, IMVR, Center for Health Services Research Cologne (ZVFK), Faculty of Medicine (FM), FHS, UoC; Prof. Dr. Ludwig Kuntz, Department of Business Administration and Health Care Management, Faculty of Management, Economics and Social Sciences (FMESS), UoC; Prof. Dr. Christian Rietz, Department of Remedial Education, FHS, UoC; Peter Ihle and Dr. Ingrid Schubert, PMV research group, FM, UoC; Prof. Dr. Stephanie Stock, Institute for Health Economics and Clinical Epidemiology, FM, University Hospital Cologne (UHC); Dr. Dr. Julia Strupp, Department of Palliative Medicine, FM, UHC; Prof. Dr. Raymond Voltz, Department of Palliative Medicine, FM, UHC.

\section{Funding}

MenDis-CHD is a sub project of the project CoRe-Net (Cologne Care Research and Development Network: An interdisciplinary learning network towards value-based care for vulnerable patients), funded by the BMBF (Federal Ministry of Research and Education, funding number: 01GY1606).

\section{Availability of data and materials}

All collected data will be load within CoRe-Net and saved into the CoRe-Net position of trust, which guaranteed a secure data storage. The CoRe-Net position of trust will allocate anonymous data for the researcher.

\section{Authors' contributions}

SP wrote the abstract, parts of the introduction, complete method section except the sub part 'qualitative' in 'materials', designed the figures, made an overall adaption of the protocol text and included references. SP was a major contributor in writing the manuscript. HM also wrote on the abstract, parts of the introduction, the discussion, made an overall adaption of the protocol text and included references in EndNote. HM was a major contributor in writing the manuscript. UK and FSN wrote the part 'qualitative' in 'materials' and the part 'content analysis 'in 'analysis.'KB wrote the part on routine data analysis. FJ and CA designed and conducted the study as chief scientists and approved the final manuscript. All authors read and approved the final manuscript.

\section{Ethics approval and consent to participate}

MenDis-CHD was approved by the Ethics Commission of Cologne University's Faculty of Medicine (committee's reference number: 17-220) on September 26th, 2017. The ethics approval for MenDis-CHD is valid for all of the seven recruitment facilities (please refer to 'procedures' for further information). The German Medical Association approved that if the researchers from MenDisCHD-team recruit the patients in the listed facilities exclusively and the physicians of these facilities do not recruit patients, there is no need for an additional ethics approval. The decision of the ethics committee of the University Hospital of Cologne is therefore sufficient for all recruitment facilities. The procedure for obtaining informed consent from the participants is as follows: when recruiting patients in cardiology departments, cardiologic rehabilitation clinics, and cardiologic practices, the first step is that the physician in charge screens his patient list for diagnoses that match our inclusion criteria. Afterward, these patients are notified to our research team.
The next step is that a member of the research team introduces him/herself to the patient and provides information about MenDis-CHD. Apart from the verbal information, every patient is handed out an information sheet, which contains details about content, procedure, and purpose of the study, contact details of the researchers, if further questions arise, commuting accident insurance, and declaration of data privacy. After the patient has read the information, the researchers detail the informed consent point-by-point. If the patient agrees to participate in the study, he/she signs the informed consent with his/her name and home address. In the informed consent, the patient is also asked, if he/she allows the research team to hand out a questionnaire-package for their relatives. If the informed consent is given, the patient and/or the relative version of the questionnaire are handed out. The questionnaire-packages contain the information letter, the copy of the informed consent, the questionnaire, as well as contact details of the research team, to address further questions. In case that the addressed relatives agree to participate in the study, the relative questionnaire-packages contain a self-addressed envelope. The described procedure for the physicians is the same as for the patients except that the physicians are identified on the basis of the participating institutions and by the information provided by the patients.

\section{Consent for publication}

Not applicable.

\section{Competing interests}

The authors declare that they have no competing interests.

\section{Publisher's Note}

Springer Nature remains neutral with regard to jurisdictional claims in published maps and institutional affiliations.

\section{Author details}

'Department of Psychosomatics and Psychotherapy, University of Cologne, Faculty of Medicine and University Hospital Cologne, Kerpener Straße 62, 50937 Cologne, Germany. ${ }^{2}$ Department of Psychiatry and Psychotherapy, University of Cologne, Faculty of Medicine and University Hospital Cologne, Kerpener Straße 62, 50937 Cologne, Germany. ${ }^{3}$ Faculty of Management, Economics and Social Sciences, University of Cologne, Albertus-Magnus-Platz, 50923 Cologne, Germany. ${ }^{4}$ PMV research group, Faculty of Medicine and University Hospital Cologne, University of Cologne, Herderstraße 52, 50931 Cologne, Germany. ${ }^{5}$ German Center for Neurodegenerative Diseases (DZNE), Sigmund-Freud-Str. 27, 53127 Bonn, Germany.

Received: 16 October 2018 Accepted: 12 March 2019

Published online: 08 April 2019

\section{References}

1. Piepoli MF, Hoes AW, Agewall S, Albus C, Brotons C, Catapano AL, et al. 2016 European Guidelines on cardiovascular disease prevention in clinical practice: The Sixth Joint Task Force of the European Society of Cardiology and Other Societies on Cardiovascular Disease Prevention in Clinical Practice (constituted by representatives of 10 societies and by invited experts) Developed with the special contribution of the European Association for Cardiovascular Prevention \& Rehabilitation (EACPR). Eur Heart J. 2016;37(29): 2315-81.

2. Mozaffarian D, Benjamin EJ, Go AS, Arnett DK, Blaha MJ, Cushman M, et al. Executive summary: heart disease and stroke statistics -2015 update. Circulation. 2015:131(4):434-41.

3. O'Flaherty M, Sans-Menendez S, Capewell S, Jørgensen T. Epidemiology of atherosclerotic cardiovascular disease: scope of the problem and its determinants. ESC Textbook Prev Cardiol. 2015;1. https://doi.org/10.1093/ med/9780199656653.003.0001.

4. Gößwald A, Schienkiewitz A, Nowossadeck E, Busch M. Prävalenz von Herzinfarkt und koronarer Herzkrankheit bei Erwachsenen im Alter von 40 bis 79 Jahren in Deutschland. BundesgesundheitsblattGesundheitsforschung-Gesundheitsschutz. 2013;56(5-6):650-5.

5. Pancholy SB, Shantha GPS, Patel T, Cheskin LJ. Sex differences in short-term and long-term all-cause mortality among patients with ST-segment elevation myocardial infarction treated by primary percutaneous intervention: a meta-analysis. JAMA Intern Med. 2014;174(11):1822-30. 
6. Arora G, Bittner V. Chest pain characteristics and gender in the early diagnosis of acute myocardial infarction. Curr Cardiol Rep. 2015;17(2):5.

7. Ladwig K-H, Lederbogen F, Albus C, Angermann C, Borggrefe M, Fischer D, et al. Position paper on the importance of psychosocial factors in cardiology: update 2013. GMS Ger Med Sci. 2014;12.

8. Barnes DE, Alexopoulos GS, Lopez OL, Williamson JD, Yaffe K. Depressive symptoms, vascular disease, and mild cognitive impairment: findings from the cardiovascular health study. Arch Gen Psychiatry. 2006;63(3):273-9.

9. Albus C, Ladwig KH, Herrmann-Lingen C. Psychocardiology: clinically relevant recommendations regarding selected cardiovascular diseases. Dtsch Med Wochenschr (1946). 2014;139(12):596-601.

10. Gohlke H, Albus C, Bönner G, Darius H, Eckert S, Gohlke-Bärwolf C, et al. Recommendations of the project group prevention of the German cardiac society (DGK) on risk adjusted prevention of cardiovascular diseases. Part 4 platelet function inhibitors, hormone replacement therapy, behavioural and psychosocial risk factors. Kardiologe. 2013;7(4):297-306.

11. Fan Z, Wu Y, Shen J, Ji T, Zhan R. Schizophrenia and the risk of cardiovascular diseases: a meta-analysis of thirteen cohort studies. J Psychiatr Res. 2013;47(11):1549-56.

12. Lichtman JH, Froelicher ES, Blumenthal JA, Carney RM, Doering LV, Frasure-Smith $\mathrm{N}$, et al. Depression as a risk factor for poor prognosis among patients with acute coronary syndrome: systematic review and recommendations. Circulation. 2014. https://doi.org/10.1161/CIR. 0000000000000019

13. Albus C. Psychosocial risk factors: time for action in the lifelong prevention of coronary heart disease. Eur J Prev Cardiol. 2017;24(13):1369-70.

14. Pogosova N, Kotseva K, De Bacquer D, von Känel R, De Smedt D, Bruthans J, et al. Psychosocial risk factors in relation to other cardiovascular risk factors in coronary heart disease: results from the EUROASPIRE IV survey. A registry from the European Society of Cardiology. Eur J Prev Cardiol. 2017;24(13):1371-80.

15. Pogosova N, Saner H, Pedersen SS, Cupples ME, McGee H, Höfer S, et al. Psychosocial aspects in cardiac rehabilitation: from theory to practice. A position paper from the cardiac rehabilitation section of the European Association of Cardiovascular Prevention and Rehabilitation of the European Society of Cardiology. Eur J Prev Cardiol. 2015;22(10):1290-306.

16. Karbach U, Schubert I, Hagemeister J, Ernstmann N, Pfaff H, Höpp H-W. Ärztliches Leitlinienwissen und die Leitliniennähe hausärztlicher Therapien. Dtsch Arztebl. 2011;108:61-9.

17. Feinstein RE, Blumenfield M, Orlowski B, Frishman WH, Ovanessian S. A national survey of cardiovascular physicians' beliefs and clinical care practices when diagnosing and treating depression in patients with cardiovascular disease. Cardiol Rev. 2006;14(4):164-9.

18. Thombs BD, Roseman M, Coyne JC, de Jonge P, Delisle VC, Arthurs E, et al. Does evidence support the American Heart Association's recommendation to screen patients for depression in cardiovascular care? An updated systematic review. PLoS One. 2013;8(1):e52654.

19. DIMDI. German Institute of Medical Documentation and Information. Health Care Data [Available from: https://www.dimdi.de/static/en/ versorgungsdaten/index.htm.

20. Porter ME. Value-based health care delivery. Ann Surg. 2008;248(4):503-9.

21. Porter ME. A strategy for health care reform - toward a value-based system. N Engl J Med. 2009;361(2):109-12.

22. Porter ME. What is value in health care? N Engl J Med. 2010;363(26): 2477-81.

23. Karbach U, Ansmann L, Scholten N, Pfaff H. Report from an ongoing research project: the Cologne Research and Development network (CoRenet) and the value-based approach to health care. Z Evid Fortbild Qual Gesundhwes. 2018;130:21-6.

24. Curtis JP, Sokol SI, Wang Y, Rathore SS, Ko DT, Jadbabaie F, et al. The association of left ventricular ejection fraction, mortality, and cause of death in stable outpatients with heart failure. J Am Coll Cardiol. 2003:42(4):736-42.

25. Johnson RB, Onwuegbuzie AJ, Turner LA. Toward a definition of mixed methods research. J Mixed Methods Res. 2007;1(2):112-33.

26. Zigmond AS, Snaith RP. The hospital anxiety and depression scale. Acta Psychiatr Scand. 1983;67(6):361-70.

27. Herrmann C. International experiences with the hospital anxiety and depression scale-a review of validation data and clinical results. J Psychosom Res. 1997:42(1):17-41.

28. Petermann F. Hospital Anxiety and Depression Scale, Deutsche Version (HADS-D). Zeitschrift für Psychiatrie, Psychologie und Psychotherapie. Göttingen: Hogrefe Verlag; 2015.
29. Wittchen H, Wunderlich U, Gruschwitz S, Zaudig M. Skid-i. Strukturiertes klinisches Interview für DSM-IV; 1997.

30. American Psychiatric Association. Diagnostic and statistical manual of mental disorders (4th ed., text rev.). 2000. https://doi.org/10.1176/appi.books. 9780890423349

31. Zanarini MC, Skodol AE, Bender D, Dolan R, Sanislow C, Schaefer E, et al. The collaborative longitudinal personality disorders study: reliability of axis I and II diagnoses. J Personal Disord. 2000;14(4):291-9.

32. Nordgaard J, Revsbech R, SæBYE D, Parnas J. Assessing the diagnostic validity of a structured psychiatric interview in a first-admission hospital sample. World Psychiatry. 2012;11(3):181-5.

33. Kalbe E, Kessler J, Calabrese P, Smith R, Passmore A. Brand ma, et al. DemTect: a new, sensitive cognitive screening test to support the diagnosis of mild cognitive impairment and early dementia. Int J Geriatr Psychiatry. 2004:19(2):136-43.

34. Kessler J, Calabrese P, Kalbe E, Berger F. DemTect: a new screening method to support diagnosis of dementia. Psycho. 2000;26(6):343-7.

35. Fillenbaum GG, van Belle G, Morris JC, Mohs RC, Mirra SS, Davis PC, et al. Consortium to establish a registry for Alzheimer's disease (CERAD): the first twenty years. Alzheimers Dement. 2008;4(2):96-109.

36. Folstein MF, Folstein SE, McHugh PR. "Mini-mental state": a practical method for grading the cognitive state of patients for the clinician. J Psychiatr Res. 1975;12(3):189-98.

37. Tombaugh TN, Mclntyre NJ. The mini-mental state examination: a comprehensive review. J Am Geriatr Soc. 1992;40(9):922-35.

38. Der PM. Mini-Mental-Status-Test (MMST) als Demenz-Screening. ZFAZeitschrift für Allgemeinmedizin. 2005;81(05):203-4.

39. Pfeffer R, Kurosaki T, Harrah C Jr, Chance J, Filos S. Measurement of functional activities in older adults in the community. J Gerontol. 1982; 37(3):323-9.

40. Teng E, Becker BW, Woo E, Knopman DS, Cummings JL, Lu PH. Utility of the functional activities questionnaire for distinguishing mild cognitive impairment from very mild Alzheimer's disease. Alzheimer Dis Assoc Disord. 2010;24(4):348.

41. Glasgow RE, Wagner EH, Schaefer J, Mahoney LD, Reid RJ, Greene SM. Development and validation of the patient assessment of chronic illness care (PACIC). Med Care. 2005;43(5):436-44.

42. Szende A, Oppe M, Devlin N. EQ-5D value sets: inventory, comparative review and user guide: Springer Science \& Business Media; 2007.

43. Graf von der Schulenburg J-M, Claes C, Greiner W, Uber A. Die deutsche Version des EuroQol-Fragebogens. Zeitschrift für Gesundheitswissenschaften= Journal of public health. 1998;6(1):3-20.

44. Schubert I, Ihle P, Köster I. Interne Validierung von Diagnosen in GKVRoutinedaten: Konzeption mit Beispielen und Falldefinition. Das Gesundheitswesen. 2010;72(06):316-22.

45. Schubert I, Köster I, Küpper-Nybelen J, Ihle P. Versorgungsforschung mit GKV-Routinedaten. Bundesgesundheitsblatt-GesundheitsforschungGesundheitsschutz. 2008:51(10):1095-105.

46. Morgan DL. Focus groups as qualitative research: sage publications; 1996.

47. Arnet I, Abraham I, Messerli M, Hersberger KE. A method for calculating adherence to polypharmacy from dispensing data records. Int J Clin Pharm. 2014:36(1):192-201.

48. Krueger K, Griese-Mammen N, Schubert I, Kieble M, Botermann L, Laufs U, et al. In search of a standard when analyzing medication adherence in patients with heart failure using claims data: a systematic review. Heart Fail Rev. 2018;23(1):63-71.

49. Gothe H, Ihle P, Swart E, Matusiewicz D. Routinedaten im Gesundheitswesen: Handbuch Sekundärdatenanalyse: Grundlagen. Huber Hans: Methoden und Perspektiven; 2014.

50. Rosenthal G. Biographical research. In: Seale C, Gobo G, Gubrium JF, David, Silverman, editors. Qualitative research practice. London: Sage; 2004.

51. Amin AA, Jones AM, Nugent K, Rumsfeld JS, Spertus JA. The prevalence of unrecognized depression in patients with acute coronary syndrome. Am Heart J. 2006:152(5):928-34.

52. Hurley MC, Arthur HM, Chessex C, Oh P, Turk-Adawi K, Grace SL. Burden, screening, and treatment of depressive and anxious symptoms among women referred to cardiac rehabilitation: a prospective study. BMC Womens Health. 2017:17(1):11. 